AIAA-98-1938

\title{
METHODS AND TECHNIQUES FOR RISK PREDICTION OF SPACE SHUTTLE UPGRADES
}

\author{
Chad R. Hoffman \& Rich Pugh \\ Pratt \& Whitney \\ West Palm Beach, FL
}

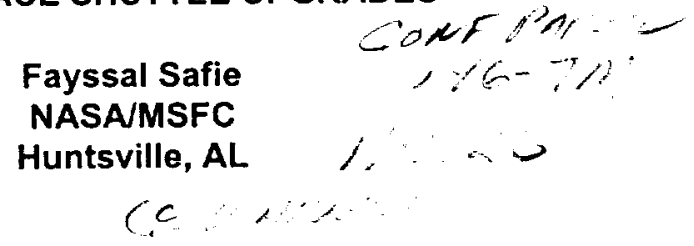

can be performed to introduce more flexibility into the design process of new hardware.

The two most commonly used methods of obtaining redesign reliability are probabilistic structural analysis and similarity analysis. ${ }^{1}$ The focus of this paper will be on probabilistic structural analysis. However, similarity analysis will be discussed briefly, and an example of similarity analysis will be provided.

\section{Similarity Method Reliability Predictions}

There are many methods and databases available to perform risk predictions using similarity analysis. Some of these methods are based on generic data such as MIL-STD-217 (used for electronic components) and NPRD95 (used for nonelectronic components) while others are based on actual data. ${ }^{2}$ Similarity analysis based on actual data will be discussed in this paper.

\section{Similarity Method Requirements}

The following requirements should be taken into account to perform effective reliability predictions:

1. The predictions must be established within the concept phase of the design.

Redesigned components, subsystems, and systems are common in the Space Shuttle program as technology increases and as unforeseen problems arise. It is desirable to be able to accurately quantify the reliability of the redesigned component as well as the subsystem and system. With the case of the Space Shuttle, it can be seen how changing the reliability of a single component affects the overall reliability of the Space Shuttle. This can be very useful information when trying to determine which components to redesign. For example, two redesigned components both may affect the overall system reliability equally, but the cost of redesigning those two components may be considerably different. Therefore, an accurate redesign reliability system can be beneficial when allocating funds for various redesign tasks. Cost and reliability trade studies
2. The most similar component must be used as the baseline.

3. All applicable historical data should be used.

4. The criticality category for each failure mode must be established and used.

The predictions should be initiated within the concept phase in coordination with the FMEA (Failure Moder.Effects Analysis). The goal of the predictions, as with all reliability tasks, is to improve the reliability of the proposed design. The predictions will assist the reliability engineer and the designer in identifying the reliability concerns that have the greatest impact on the reliability of the product. Obviously, in an ideal world all the 
reliability concerns should be addressed, but in the real world, money and schedules must be taken into account. Properly performed predictions provide the means to address the "big hitters".

Another important factor in establishing the predictions early in the design process is to use them during the trade studies. Design concepts may be traded very early within the design process. Therefore, to have an impact, the predictions, or at least baselines, must be in place to quantify the reliability concerns during the trades.

The reliability of the most similar component should be used as the baseline. Predictions must take into account similarities as well as differences between the baseline design and operational environment. By choosing a baseline component that is most similar to the proposed design, fewer variables are present which can impact the accuracy of the predictions.

All applicable data must be used in developing the predictions. In the rocket engine area, many believe only flight data should be used in establishing the reliability of the engines. However, ground testing may provide an even better indication of the true reliability, and the combination of the two provides a more complete picture. Obviously, the data have to be properly screened to eliminate data that are due to ground firings that do not represent the true design or realistic environments. Thus very early development designs and testing to extreme environments should be eliminated. The criticality category of each failure should be taken into account as part of the baseline assessment and reliability predictions. There is usually confusion with the reliability categories to anyone not familiar with them. When asked what is the reliability of a product, the answer should be: "Which reliability?".

\section{Why Perform the Reliability Predictions?}

Reliability engineers are frequently asked: "Why should we bother spending the time and money in performing predictions?"; "What good are they?", "How will they make the product better?"; etc. The following provide a list of good answers:

1. Allows the prioritizing of the high risk failure modes (more "bang for the buck" in the design).
2. Obligates the design engineers to consider reliability equally with the other system parameters such as weight, cost, and performance.

3. Provides guidance in the selection of design concepts through trade studies.

4. Assists in quantifying the effects of design variability.

5. Provides an early indication of meeting reliability goals.

6. Enhances the effectiveness of the development test program.

7. Provides input to the Life Cycle Cost Model.

8. Establishes both scheduled and unscheduled maintenance requirements.

9. Provides input to the Logistics Support Analysis.

10. Cuts unscheduled maintenance time to repair by allowing the design to accommodate the most unreliable components.

The bottom line is that if timely predictions are performed, the impact on the design and subsequent operating costs can be enormously beneficial to the product.

\section{Similarity Method Prediction Example}

To show how this method works in developing a reliability prediction, consider a fuel turbopump example. The task is to estimate the reliability of a new turbopump design. The first step is to establish the historical data for the reliability baseline. This number is derived using the binomial method with a $90 \%$ statistical confidence. This high level of confidence is necessary to ensure the baseline has a high level of accuracy. Assume the turbopump failure rate is 50 failures per 100,000 engine firings. Now the distribution of the piece part failures is considered to identify the big hitters needing improvement.

Having identified the turbine blade as the piece part with the highest failure rate, the causes of failures must now be identified in order to identify and quantify the potential fixes. The turbine blades were the number one contributor with a percentage 
of $35 \%$ or 17.5 failures per 100,000 engine firings. Let's further assume that the distribution for the turbine blades shows that the number one cause is thermal stresses which have $57 \%$ of the total blade failures or a rate of 10 per 100,000 firings.

Next, the new design is evaluated and the baseline failure rates are adjusted. These adjustments, in order of preference, are established through testing, analyses, or expert opinion (Delphi approach). Returning to the example, and addressing the "heavy hitters" of thermal stresses, the proposed design is assumed to have modified the operating temperatures, incorporated a hollow blade design, and incorporated a material change. Using the adjustment for each of these changes, the cumulative failure rate adjustment is 5.52 per 100,000 engine firings. Therefore, the fuel turbopump failure rate for the proposed design, if no other changes are made, drops from the original 50 to 44.48 failures per 100,000 firings.

\section{Actual Similarity Method Prediction Example}

This method has been successfully used on previous jet engine programs. In the early 1980's, the Air Force wanted a jet fighter engine with increased reliability and lower maintenance requirements to decrease life cycle costs. A new engine design and development program was initiated, and the reliability predictions were established using the previous engine as the baseline. These predictions were made approximately 54 months prior to operation.

As with the previous example, the components were assessed at the part and failure mode levels. When the analysis was complete, the predicted failure rate of the new engine was approximately $25 \%$ of the previous engine's failure rate. This dramatic increase in reliability was due to a very effective use of lessons learned and reliability data.

When the total operating time of the new engine reached the point $\left(T_{0}\right)$ where the prediction was based, a comparison of the actual failure rate versus the predicted failure rate was made. A difference of only $4 \%$ existed between the predicted value and the actual value. Figure 1 shows the previous actuals, the point of estimate, and the delta between the estimate and the new actuals.

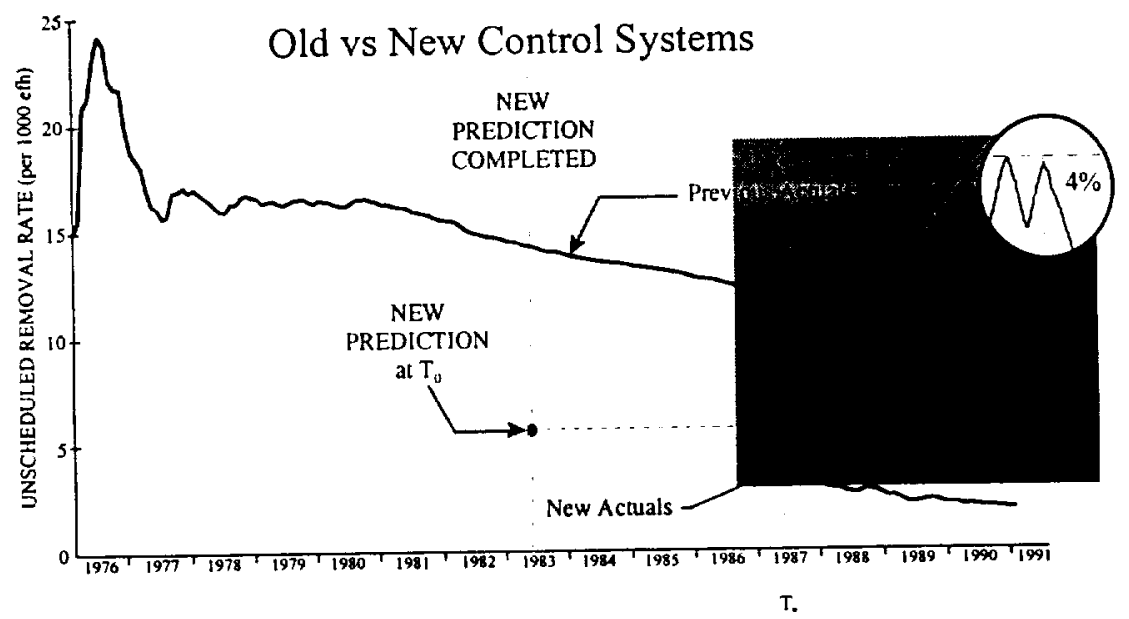

Prediction within $4 \%$ of actual.

Figure 1 Similarity predictions can provide a high degree of accuracy

\section{Probabilistic Structural Reliability Predictions}

Unlike similarity analysis, probabilistic structural analysis uses the actual design structural failure mode model to calculate reliability predictions. ${ }^{3}$ These predictions are not based on similar components and past test experience. They are based on the structural model and the variation of the parameters or input variables in the structural model. Probabilistic design methodology considers statistical distributions of the lifecontrolling variables thus providing a distribution of component reliability. Probabilistic design 
methodology must be integrated within an organization's current design system. ${ }^{4}$

Monte Carlo simulation (where feasible) is the most accurate probabilistic methodology as the sample size increases to infinity. Monte Carlo simulation entails characterizing each input or lifecontrolling variable with a distribution and then simulating from this distribution a large number of times. This large number of combinations of simulated random variables is then run through the life equation or design code and a large number of output or life variables is obtained. A distribution can then be fit to the output or life variable which then can be evaluated at the desired point of interest (failure criterion). This evaluation will determine the reliability of the component. From this reliability number, future failures can be predicted over the course of the life of the component. A typical Monte Carlo simulation flowchart is shown in Figure 2.

Some design codes are very complex and timeconsuming thus prohibiting thousands of Monte Carlo Simulations. In these cases, alternative methods are applied that use fewer design-codes runs to approximate the "true" structural models. An example of this is a Response Surface Monte Carlo simulator where a Box Behnken or Central Composite designed experiment is used to systematically make a small number of design code runs in order to fit a response surface to the output data points. ${ }^{5}$ A Box Behnken design (See Figure 3 ) is a three-level design that is used to fit response surfaces. Points in the design space are systematically chosen for each random variable ( $\mu$ $k \sigma, \mu, \mu+k \sigma$ ) where $k$ is a constant such that all main effects, 2-way interactions, and $2^{\text {nd }}$ order terms can be estimated (See Table 1) ${ }^{6}$

A Central Composite design (See Figure 4) is also a $2^{\text {nd }}$ order design that is a factorial or fractional factorial design with the addition of center points and star points. This design therefore has five levels $(\mu-k \sigma,-\alpha, \mu, \alpha, \mu+k \sigma)$ where $\alpha$ is the "star" point that is chosen to allow estimation of the $2^{\text {nd }}$ order terms (See Table 2). ${ }^{7}$ Unless a response surface is highly nonlinear, Box-Behnken and Central Composite designs usually estimate the "true" response surface very accurately.

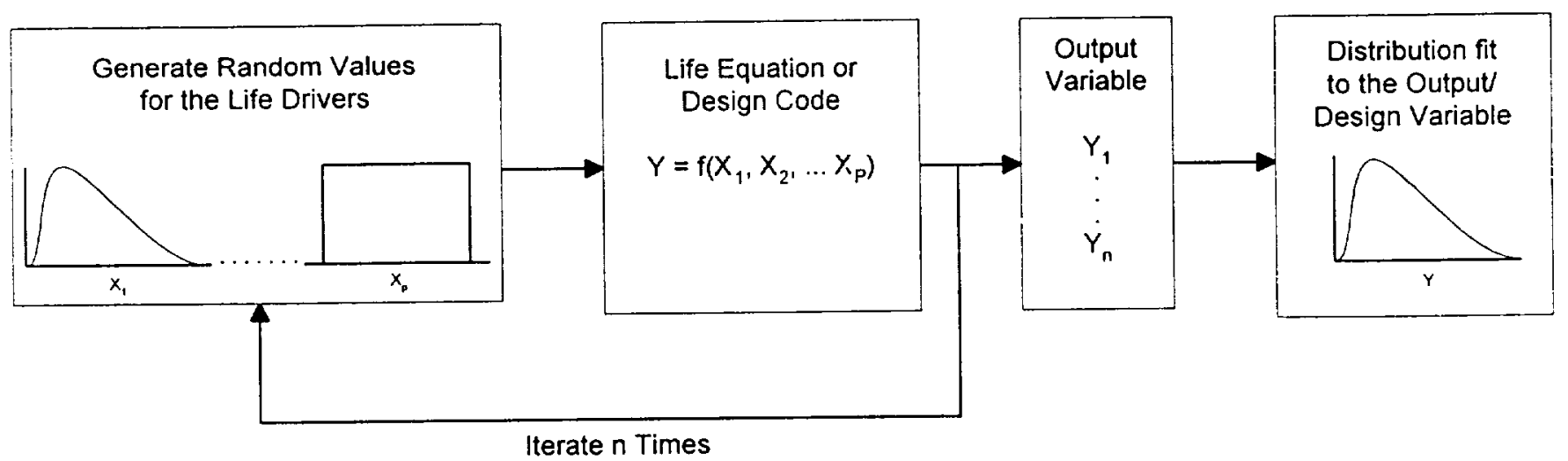

Figure 2 Monte Carlo Flowchart

Table 1 A Three-Variable Box-Behnken Design

\begin{tabular}{cccc}
\hline Run & $x_{1}$ & $x_{2}$ & $x_{3}$ \\
\hline 1 & -1 & -1 & 0 \\
2 & -1 & 1 & 0 \\
3 & 1 & -1 & 0 \\
4 & 1 & 1 & 0 \\
5 & -1 & 0 & -1 \\
\hline
\end{tabular}

\begin{tabular}{cccc}
\hline 6 & -1 & 0 & 1 \\
7 & 1 & 0 & -1 \\
8 & 1 & 0 & 1 \\
9 & 0 & -1 & -1 \\
10 & 0 & -1 & 1 \\
11 & 0 & 1 & -1 \\
12 & 0 & 1 & 1 \\
13 & 0 & 0 & 0 \\
14 & 0 & 0 & 0 \\
15 & 0 & 0 & 0 \\
\hline
\end{tabular}


AIAA-98-1938

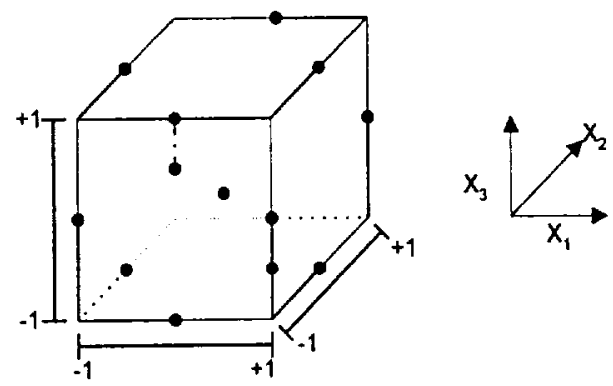

Figure 3 A Three Variable Box-Behnken Design

Table 2 A Three-Variable Central Composite Design

\begin{tabular}{cccc}
\hline Run & $x_{1}$ & $x_{2}$ & $x_{3}$ \\
\hline 1 & -1 & -1 & -1 \\
2 & -1 & -1 & 1 \\
3 & -1 & 1 & -1 \\
4 & -1 & 1 & 1 \\
5 & 1 & -1 & -1 \\
6 & 1 & -1 & 1 \\
7 & 1 & 1 & -1 \\
8 & 1 & 1 & 1 \\
9 & $-\alpha$ & 0 & 0 \\
10 & $\alpha$ & 0 & 0 \\
11 & 0 & $\alpha$ & 0 \\
12 & 0 & $-\alpha$ & 0 \\
13 & 0 & 0 & $-\alpha$ \\
14 & 0 & 0 & $\alpha$ \\
15 & 0 & 0 & 0 \\
16 & 0 & 0 & 0 \\
17 & 0 & 0 & 0 \\
\hline
\end{tabular}

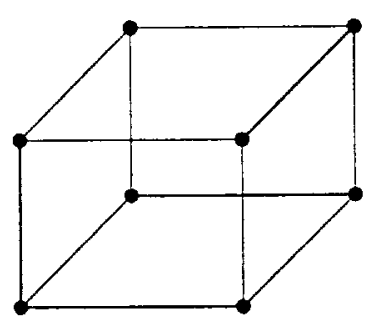

a.) Cube Portion $( \pm 1, \pm 1, \pm 1)$ b.) "Star" Portion

$$
\begin{aligned}
& ( \pm \alpha, 0,0) \\
& (0, \pm \alpha, 0) \\
& (0,0, \pm \alpha)
\end{aligned}
$$

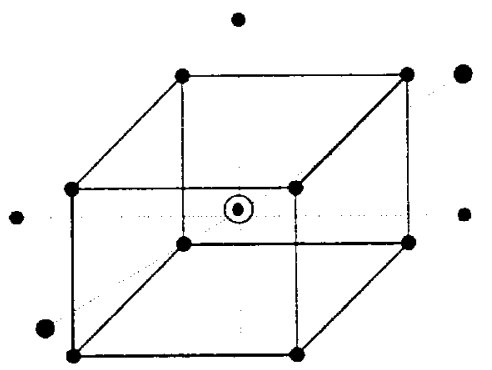

c.) Center Points $(0,0,0)$

d.) Cube + Star + Center

$( \pm 1, \pm 1, \pm 1)$,

$( \pm \alpha, 0,0)$

$(0, \pm \alpha, 0)$

$(0,0, \pm \alpha)$

$(0,0,0)$

Figure 4 A Central Composite Design for Three Variables

\begin{tabular}{|ccccc|}
\hline \multicolumn{5}{|c|}{ Box-Behnken Matrix } \\
\multicolumn{7}{|c}{$x_{1}$} & $x_{2}$ & & $x_{p}$ \\
1 & -1 & 0 & $\ldots$ & -1 \\
2 & -1 & 0 & $\cdots$ & +1 \\
3 & +1 & 0 & $\cdots$ & -1 \\
4 & +1 & 0 & $\cdots$ & +1 \\
. &. &. & $\cdots$ &. \\
. &. &. & $\cdots$ & + \\
$n$ & 0 & -1 & $\cdots$ & +1 \\
\hline
\end{tabular}

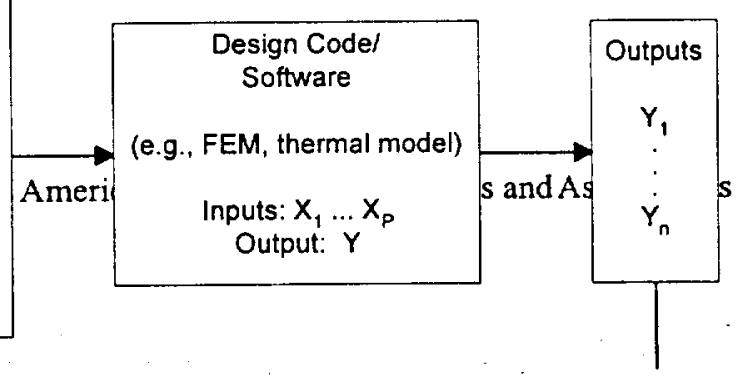


AIAA-98-1938
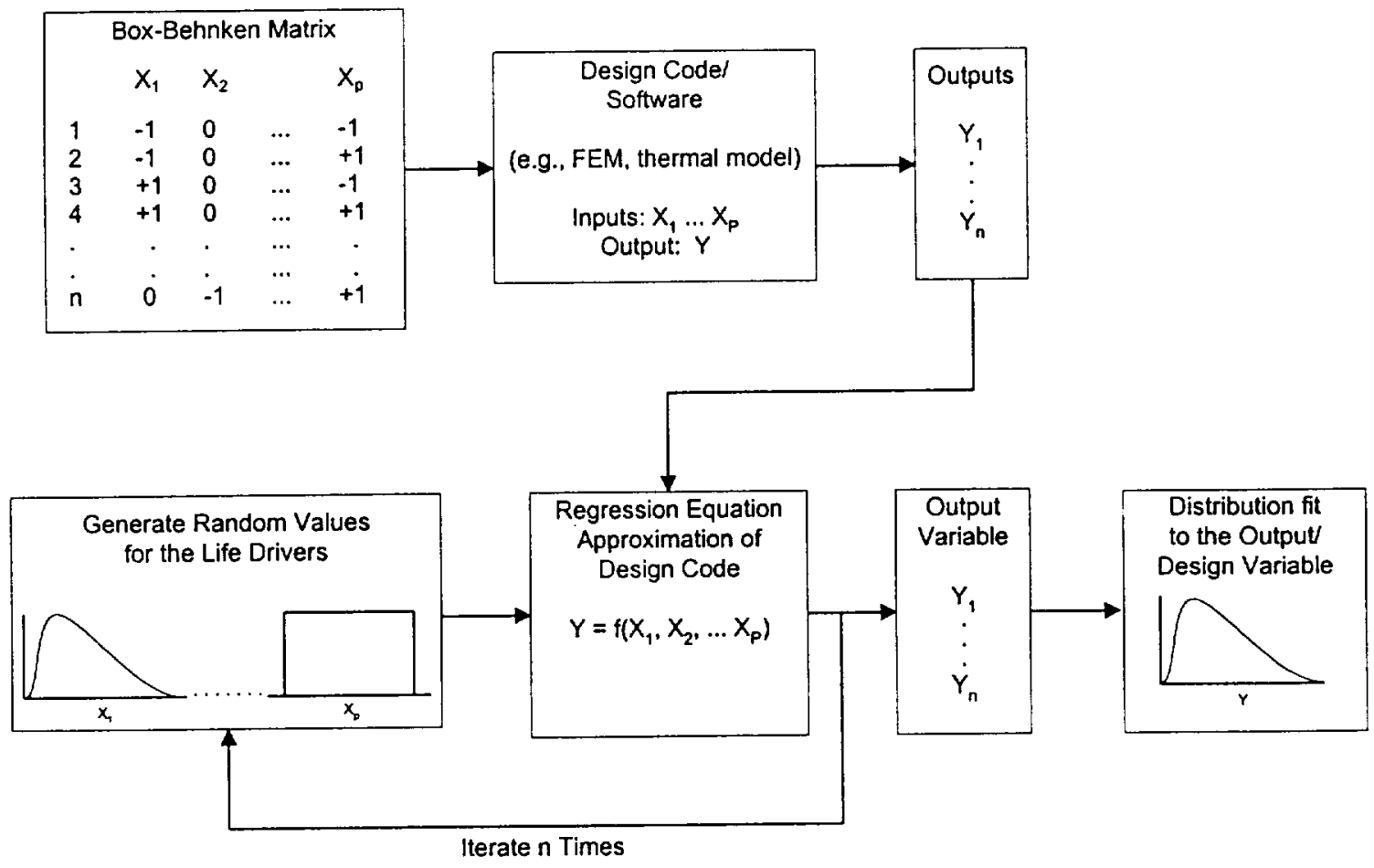

Figure 5 Response Surface Monte Carlo Flowchart

The complex design code is therefore approximated with a response surface regression model and a Monte Carlo simulator can be run using this regression model. The large number of iterations using this regression model will be much faster than using the complex design code. Finally, a distribution can be fit to the output or life variable and this distribution can be evaluated where desired. A flowchart of the Response Surface Monte Carlo is shown in Figure 5.

Several other probabilistic methodologies exist when Monte Carlo cannot be used due to time and/or cost constraints. These methods all attempt to maintain high accuracy while saving considerable time in the reliability analysis. Response Surface Monte Carlo as previously described is heavily dependent on being able to find an accurate yet simplified approximation to the complex design code. The response surface model can be checked for goodness of fit by checking several criteria $\left(R^{2}\right.$, residual plots, $p$ values, influential points, etc.). If the fitted response surface model "passes" all of the criteria for a good fitting model, then it would seem reasonable to use this approximated model in the Monte Carlo Simulation. ${ }^{8}$

In addition to Response Surface Monte Carlo simulation, there are many other probabilistic numerical methods used for calculating component reliability. These methods can be grouped into approximately five common groups of probabilistic methods. The first common group is simulation methods which contains Monte Carlo Simulation, Directional Simulation, and Latin Hypercube Simulation. These methods are usually the most accurate (especially Monte Carlo Simulation) but are also often time consuming and computer intensive. The second group is response surface/designed experiments which contains Response Surface Monte Carlo using a Box Behnken, Central Composite, or a variety of other designed experiments. These methods are very accurate if the design code or life equation can be accurately estimated with a response surface model. The third group of probabilistic methods includes First Order Reliability Method 
(FORM), Second Order Reliability Method (SORM), and Fast Probability Integration (FPI). The accuracy of these methods decreases as the number of random variables increases. The fourth group of probabilistic methods is importance sampling which includes the Importance Sampling Method using factor and radius (ISAMF \& ISAMR), Adaptive Importance Sampling $1^{\text {st }}$ Order (AIS1), and Adaptive Importance Sampling $2^{\text {nd }}$ Order (AIS2). These methods sample around the failure region rather than the entire sample space, thus saving sampling and simulation time. Finally, the fifth group of probabilistic methods falls into the mean-based methods category and includes the Advanced Mean Value Method (AMV), and the Advanced Mean Value plus iterations (AMV+). These methods utilize point expansion and perturbation techniques to estimate the failure probability. ${ }^{9}$

Probabilistic structural analysis makes use of structural models to calculate a failure mode distribution. Each input variable (temperature, stress, etc.) is considered as a random variable. These variables are then simulated from a historically-based or assumed distribution and input into the structural model. This process is repeated a large number of times to produce a failure mode distribution. This distribution can be evaluated at various points to determine a probability of failure (i.e. cumulative failure percent by 60 missions).

Various failure modes such as low cycle fatigue (LCF), high cycle fatigue (HCF), fracture life, margin of safety (M.O.S.), and thermal mechanical fatigue (TMF) can be analyzed probabilistically. As long as a structural model can be defined that predicts these failure modes and the distributions for all the input variables included in the structural model can be defined, a probabilistic analysis can be performed. Input variable distributions may come from historical data (test data) or engineering assessment. These distributions may be Beta, Exponential, Lognormal, Normal, Weibull, or any other distribution that fits the data accurately (see Figure 6). The values of the input variables (stress, temperature, etc.) are then simulated from these distributions a large number of times forming many different combinations of the input variables. These various combinations are then run through the structural model (LCF, HCF, etc.) to obtain a distribution of the output variable.

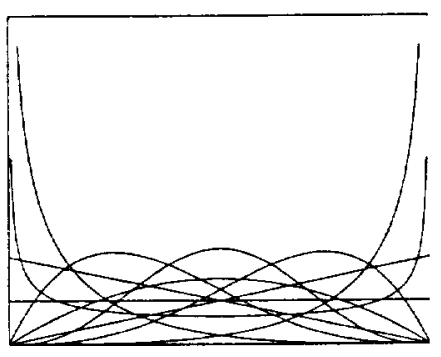

Beta

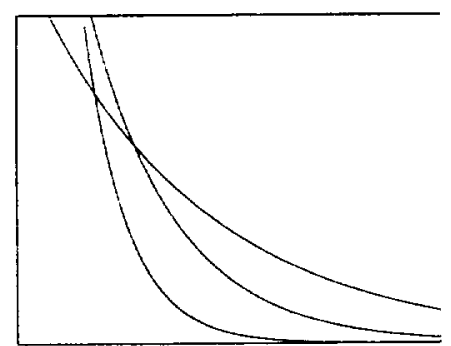

Exponential

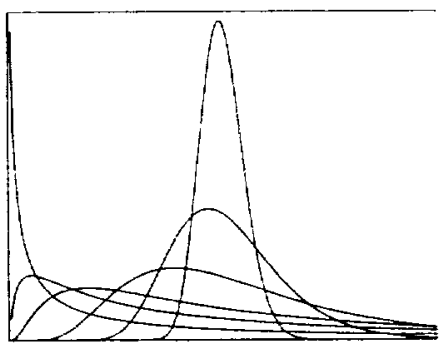

Lognormal

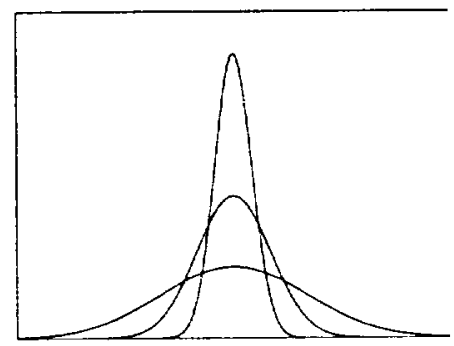

Normal

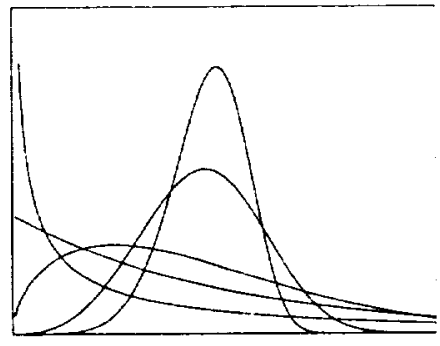

Weibull

Figure 6 Possible Shapes for the Beta, Exponential, Lognormal, Normal, and Weibull Distributions Probabilistic Structural Analysis Example 
AIAA-98-1938

A probabilistic structural analysis example of an SSME HPOTP 1-2 Turbine Spacer Margin of Safety (M.O.S.) follows:

$$
\text { M.O.S. }=\left(\frac{\sigma_{U T S}}{\sigma_{104 \%(\mathrm{nom})}}-1\right)
$$

where:

$\sigma_{\text {UTS }}=$ Ultimate Tensile Strength (KSI)

$\sigma_{104 \%(n o m)}=$ Nominal Operating Stress (KSI) for

a $104 \%$ Nominal Mission

$\sigma_{U T S}=f(T)$ where $\mathrm{T}$ is Temperature $\left({ }^{\circ} F\right)$
(M.O.S.) Ultimate. First the variables temperature and stress $\left(\sigma_{104}\right)$ are simulated from normal distributions. Then temperature is used in a regression model (with error) to predict Ultimate Tensile Strength (UTS). The resulting ultimate tensile strength and stress values are used in the Margin of Safety equation to calculate a value for Margin of Safety. This process is repeated a large of number of times and a distribution is fit to the Margin of Safety values.

Figure 7 shows the process used to obtaip START failure mode distribution for Margin of Sam

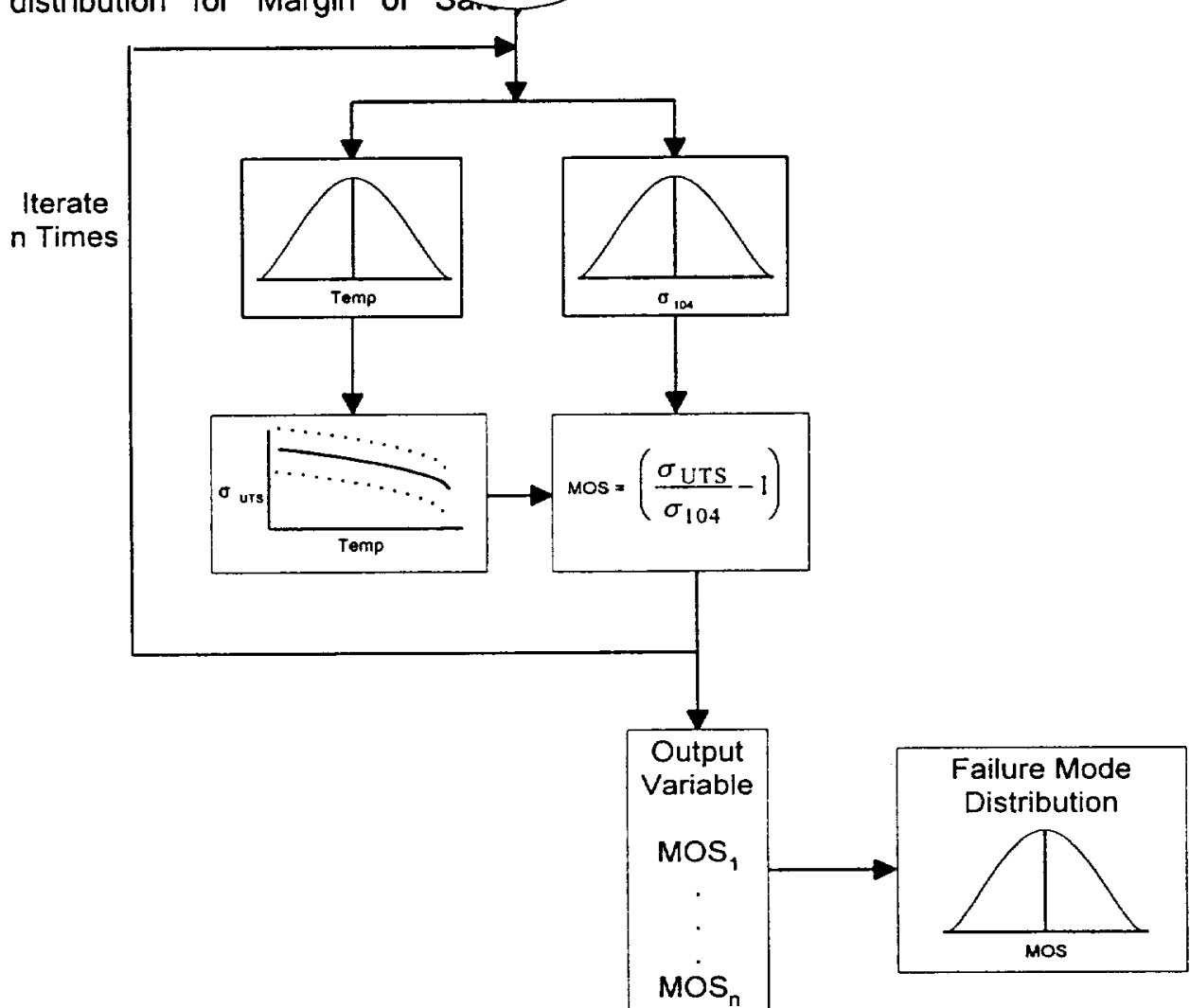

Figure 7 M.O.S. Failure Mode Distribution Flowchart

\section{Uncertainty Distributions}

8

American Institute of Aeronautics and Astronautics 
In the Probabilistic Structural Analysis Example, the input variables were simulated from normal distributions or predicted from regression models to form a distribution of margin of safety (M.O.S.). This distribution was analyzed to determine the probability of having a margin of safety less than zero (which constitutes a failure). Analyzing the resulting failure distribution for margin of safety at zero results in a single probability estimate of failure. To obtain the uncertainty about this estimate, several methods are proposed.

\section{Hyperparameterization}

Each input variable was simulated from a distribution characterized by one or more parameters. Placing variation on these parameters due to uncertainty and running a
Monte Carlo simulation with a different parameter each time would result in a large number of different margin of safety failure mode distributions when finished. Then each failure mode distribution could be analyzed at zero to obtain the probability of failure. Therefore, a distribution of failure probabilities would be obtained. For instance, instead of saying that the probability of fracturing a 1-2 Turbine Spacer due to negative margin of safety was $1.0 \times 10^{-9}$, it could now be stated that the range of failure probabilities was between $1.0 \times$ $10^{-11}$ to $1.0 \times 10^{-7}$ with $95 \%$ confidence. This method seems the most realistic since the designer/engineer may not be $100 \%$ confident of the true mean and variation of the minimum and maximum temperatures and stresses. By allowing these parameters to vary, some of the uncertainty that exists in these variables is captured.

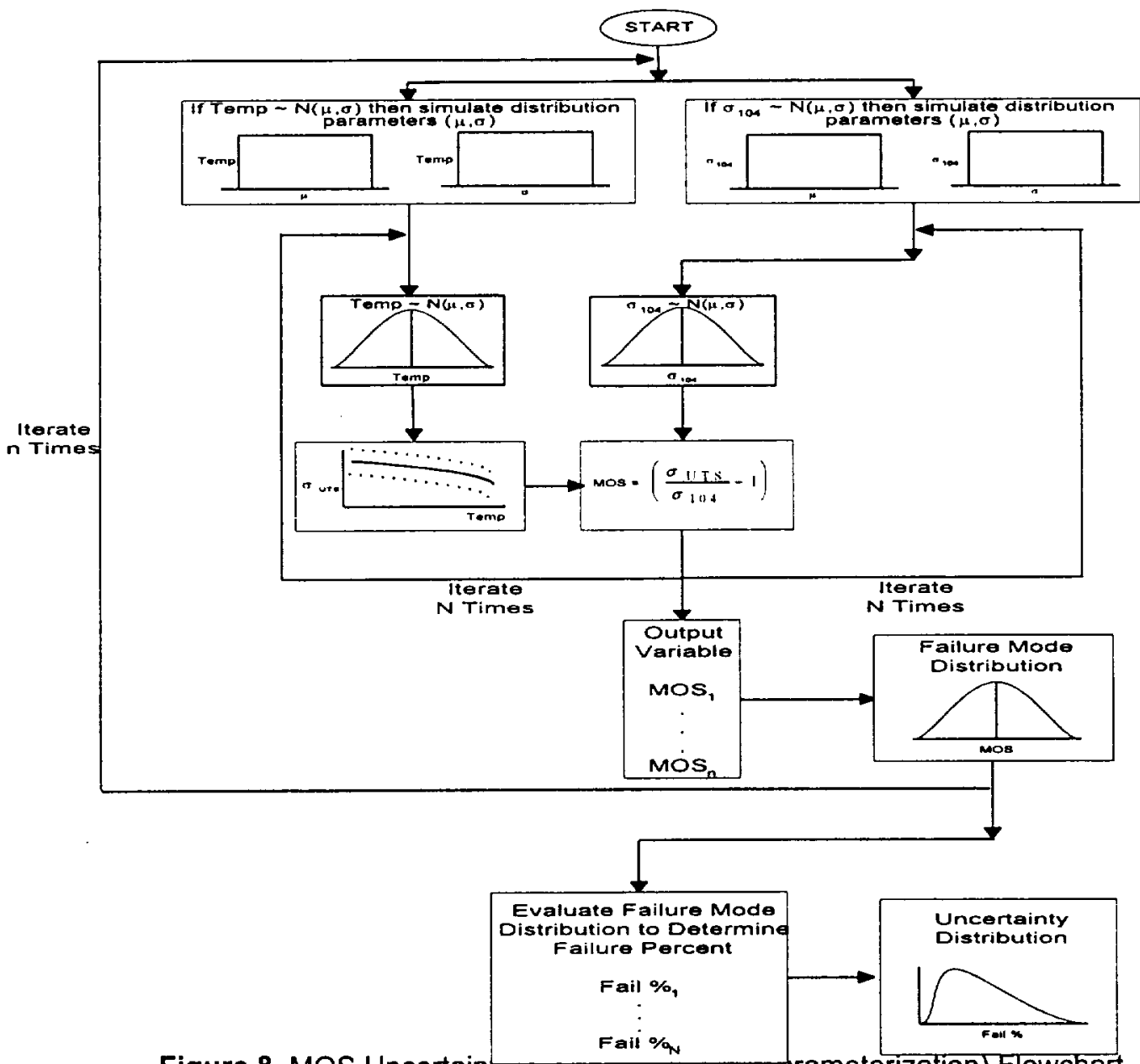

Figure 8 MOS Uncertainty DISTnDuturi (nyperparameterization) Flowchart

As shown in Figure 8 , first the normal distribution parameters $\left(\mu_{1}, \sigma_{1}\right)$ for temperature are simulated from uniform distributions. Then the normal distribution parameters $\left(\mu_{2}, \sigma_{2}\right)$ for $\sigma_{104}$ (stress at $104 \%$ operating conditions) are simulated from uniform distributions. The temperature

9

American Institute of Aeronautics and Astronautics 
parameters are then used to simulate a temperature value from a normal distribution $\left(\mu_{1}\right.$, $\left.\sigma_{1}\right)$. This value of temperature is used to predict a value of $\sigma_{\text {UTS }}$ (ultimate tensile strength). The $\sigma_{104}$ parameters are then used to simulate a $\sigma_{104}$ value from a normal distribution $\left(\mu_{2}, \sigma_{2}\right)$. These values are used to calculate a margin of safety (M.O.S.) value. Temperature and $\sigma_{104}$ values are repeatedly simulated and a distribution is fit to the M.O.S. values. This distribution is evaluated at the failure criterion to determine the probability of failure. This entire process is repeated (beginning with simulating new normal distribution parameters for both temperature and $\sigma_{104}$ ) until a distribution can be fit to the probability of failure values, thus representing the uncertainty distribution for probability of failure.

\section{Bootstrapping}

Bootstrapping would be a final possibility to obtain an uncertainty distribution. Bootstrapping, a form of resampling with replacement from a data set, is frequently used when there is a lack of data. ${ }^{10}$ The resampling produces many "pseudo" data sets from the original data set. These new "pseudo" data sets can then be analyzed to obtain a distribution of the parameters of interest.

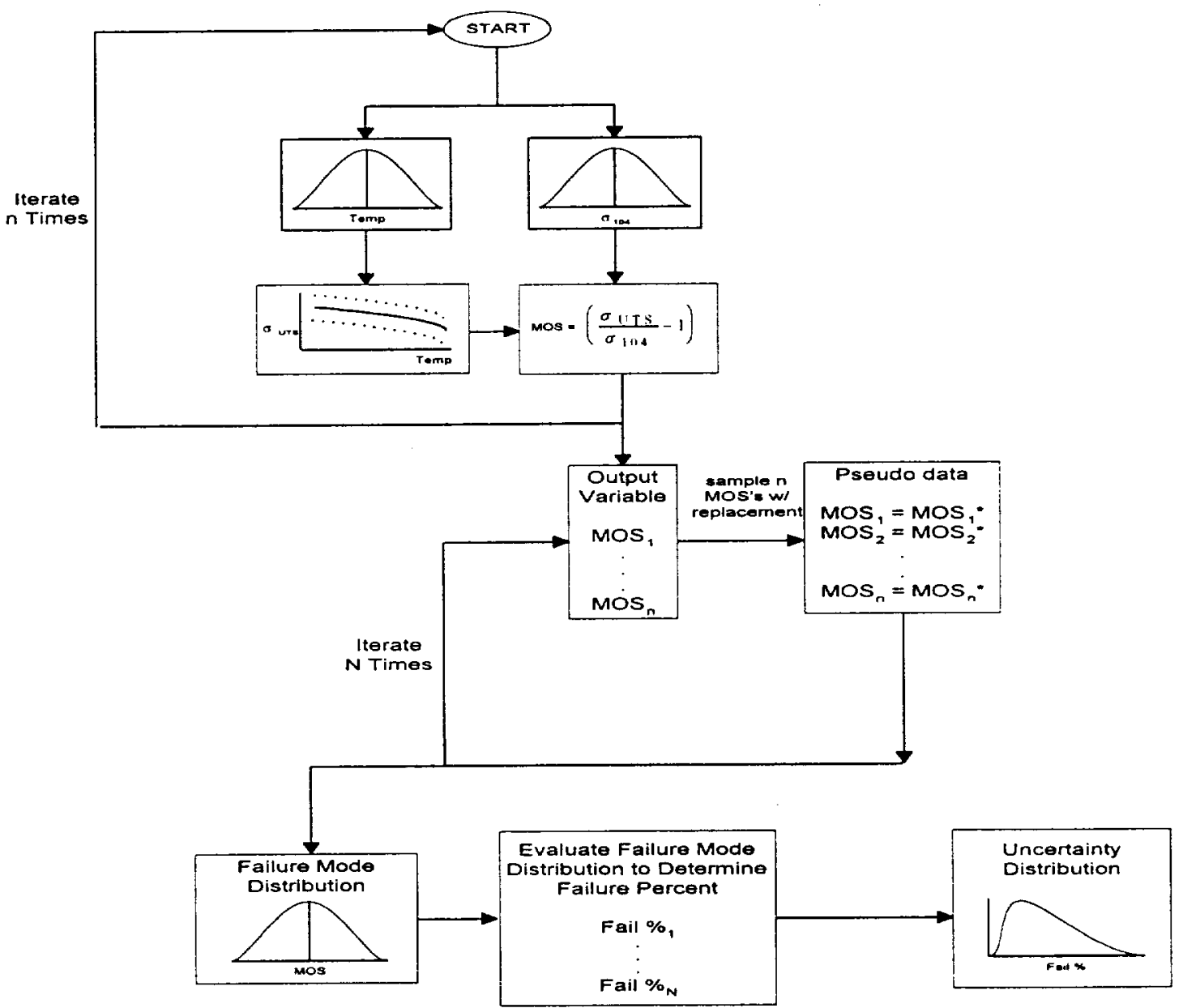

Figure 9 M.O.S. Uncertainty Distribution (Bootstrapping) Flowchart

Resampling with replacement from the failure mode distribution would produce many different failure mode distributions which could be analyzed at the desired point of interest. This method also captures the uncertainty about the original failure mode distribution. As shown in Figure 9, first, temperature and $\sigma_{104}$ are simulated from normal distributions $(\mu, \sigma)$. Then temperature is used to predict $\sigma_{\text {UTS }}$ based on a material regression model. Margin of Safety (M.O.S.) is calculated using $\sigma_{104}$ and $\sigma_{\mathrm{uts}}$. This process is repeated a large number

American Institute of Aeronautics and Astronautics 
AIAA-98-1938

of times and a distribution is fit to the Margin of Safety (M.O.S.) values.

This distribution is then evaluated at the failure criterion to determine a failure probability. Then a new sample with replacement is taken from the original simulated sample of M.O.S. values and a distribution is fit to this sample and evaluated at the failure criterion. This process of resampling from the original M.O.S. sample is repeated a large of number of times. Finally, a distribution is fit to the failure probabilities, thus forming the uncertainty distribution for the failure probabilities.

\section{Conclusions}

Probabilistic structural analysis and similarity analysis are two common analysis techniques used for assessing the reliability of redesigned hardware. Similarity analysis is used when there is a lack of data concerning the proposed redesigned component. Probabilistic structural analysis can be used when a structural model and the corresponding input variables are well defined. Monte Carlo Simulation is the most common form of probabilistic structural analysis and is the most accurate. However, sometimes Monte Carlo Simulation cannot be performed due to the complexity and time consuming nature of a particular design code. There are many other probabilistic methods that can be applied in these situations. Probabilistic structural analysis was applied to a variety of engineering problems and the resulting failure mode and uncertainty distributions were calculated. Overall, probabilistic structural analysis and similarity analysis can be a very useful tool for determining the reliability of redesigned components.

\section{References}

1. Safie, Fayssal, (1993), "A New Reliability Evaluation Approach for Rocket Engines," Industrial Engineering Research Conference, Los Angeles, CA.

2. Pugh, Rich (1996), "Reliability for the Life of the Program", 1996 Annual Reliability and Maintainability Symposium.

3. Safie, Fayssal, (1992), "Use of Probabilistic Design Methods for NASA Applications,"
ASME Symposium on Reliability Technology, Anaheim, CA.

4. Fox, Eric P. (1994), "The Pratt \& Whitney Probabilistic Design System," $35^{\text {th }}$ AIAAIASME/ASCE/AHSIASC Structures, Structural Dynamics, and Materials Conference, AIAA-94-1442, April, 1994.

5. Fox, Eric P. (1993), "Methods of Integrating Probabilistic Design Within an Organization's Design System Using Box-Behnken Matrices," $34^{\text {th }}$ AIAA/ASME/ASCE/AHS/ASC Structures, Structural Dynamics, and Materials Conference, AIAA-93-1380-CP, April 1993.

6. Montgomery (1991), Design and Analysis of Experiments, $3^{\text {rd }}$ Edition, John Wiley \& Sons, New York.

7. Box \& Draper (1987), Empirical Model-Building and Response Surfaces, John Wiley \& Sons, New York.

8. Fox, Eric P. (1996), "Issues in Utilizing Response Surface Methodologies for Accurate Probabilistic Design," American Institute of Aeronautics, Inc. April 1996.

9. Liu, Minda (1996), "Probabilistic Design Methods for Reliability Assessment," Joint Statistical Meeting, Chicago, IL, August 1996.

10. Efron, B. \& Tibshirani, R. J. (1993), An Introduction to the Bootstrap, Chapman Hall, New York. 